\title{
Evaluación ergonómica mediante el método ROSA en docentes con teletrabajo de la UTEQ, 2020
}

Ergonomic evaluation using the ROSA method in teachers with telework of the UTEQ, 2020

Jean Carlos Vallejo Morán ${ }^{1 *}$ iD

Universidad Técnica Estatal de Quevedo

Irene Teresa Bustillos Molina ${ }^{2}$ iD

Universidad Técnica Estatal de Quevedo

Eudes Martínez Porro ${ }^{*}$ (iD

Universidad Técnica Estatal de Quevedo

Ernestina Clemencia Leon Coello ${ }^{4}$ iD

Universidad Técnica Estatal de Quevedo

Fecha recepción: 15 de junio de 2020

Fecha aceptación: 16 de julio de 2020

- 2020 Universidad de Cordoba. Este es un artículo de acceso abierto distribuido bajo los términos de la licencia Creative Commons Attribution License, que permite el uso ilimitado, distribución y reproducción en cualquier medio, siempre que el autor original y la fuente se acreditan.

\footnotetext{
1 Ingeniero en Seguridad Industrial y Salud Ocupacional, Universidad Técnica Estatal de Quevedo, Graduado/Facultad Ciencias de la Ingeniería, Valencia, Ecuador, jean.vallejo2015@uteq.edu.ec, 0939645255, https://orcid.org/0000-0002-1551-8609*

2 Magister en Seguridad y Salud Ocupacional, Universidad Técnica Estatal de Quevedo, Docente/Salud Ocupacional y Seguridad en el Trabajo/Facultad Ciencias de la Ingeniería Quevedo, Ecuador, ibustillos@uteq.edu.ec, https://orcid.org/0000-0001-8647-7077

3 Magister en Salud de los Trabajadores, Universidad Técnica Estatal de Quevedo, Docente/Salud Ocupacional y Seguridad en el Trabajo/Facultad Ciencias de la Ingeniería, Quevedo, Ecuador, emartinez@uteq.edu.ec, https://orcid.org/0000-0002-6433-2173.

4 Doctor en Ciencias Pedagógicas, Universidad Técnica Estatal de Quevedo, Coordinadora de Carrera de Seguridad Industrial/Salud Ocupacional y Seguridad en el Trabajo/Facultad Ciencias de la Ingeniería, Quevedo, Ecuador, ecoello@uteq.edu.ec, https://orcid.org/0000-0002-6251-9233
} 


\section{RESUMEN}

Este proyecto de investigación se realizó con el objetivo de realizar una evaluación ergonómica de las posturas inadecuadas adoptadas por los docentes de la Universidad Técnica Estatal de Quevedo en la modalidad de teletrabajo, y luego gestionar los resultados de manera técnica. En el estudio se evaluó la muestra con dos herramientas, el método ROSA, y la encuesta ergonómica, los resultados mostraron que los docentes se encuentran afectados por riesgos ergonómicos, debido a las condiciones actuales poco favorables para realizar sus actividades diarias de teletrabajo desde sus hogares; así mismo se identificó que las partes afectadas del cuerpo de los docentes son el cuello, la espalda alta y baja lo cual se debe al desconocimiento en cuanto la ergonomía que deben tener al realizar sus actividades. Luego de realizar la evaluación respectiva a través del software Ergoniza y analizados los datos, se propone un plan de acción para capacitar, instruir y fomentar cultura en los docentes, tanto en el uso de equipos físicos de trabajo como en la adecuación del entorno de trabajo; lo cual permita mitigar y corregir los riesgos en un cierto grado. Esto conllevará a generar los controles necesarios para garantizar y precautelar la seguridad y salud de los trabajadores.

PALABRAS CLAVE: Gestión técnica, evaluación ergonómica, método ROSA, riesgos ergonómicos, plan de acción.

\section{ABSTRACT}

This research project was carried out with the objective of making an ergonomic evaluation of the inappropriate postures adopted by the teachers of the Universidad Técnica Estatal de Quevedo in the telework modality, and then manage the results in a technical way. In the study, the sample was evaluated with two tools, the ROSA method, and the ergonomic survey, the results showed that teachers are affected by ergonomic risks, due to the current unfavorable conditions to carry out their daily telework activities from their homes; Likewise, it was identified that the affected parts of the teachers' bodies are the neck, upper and lower back, which is due to ignorance regarding the ergonomics they should have. After carrying out the respective evaluation through the software Ergoniza and analyzing the data, an action plan is proposed to train teachers, both in the use of physical work equipment and in the adaptation of the work environment; which allows mitigating and correcting risks to a certain degree. This will lead to generating the necessary controls to guarantee and protect the safety and health of workers.

KEYWORDS: Technical management, ergonomic evaluation, ROSE method, ergonomic risks, action plan. 


\section{INTRODUCCIÓN}

En los actuales momentos la crisis que afecta al mundo entero, ha obligado a las instituciones a optar por nuevas modalidades de trabajo, entre ellas la modalidad virtual o teletrabajo. En esta línea de ideas las Instituciones de Educación Superior implementaron la modalidad en línea, pero en la búsqueda de alcanzar sus objetivos como instituciones se deja en ocasiones a un lado la salud del docente. La modalidad virtual conlleva a que el docente tenga que permanecer sentado realizando sus actividades por un tiempo prolongado; esta exposición tiene sus consecuencias una de ellas son los dolores en la espalda, en especial en la zona lumbar, convirtiéndose uno de los problemas laborales más frecuentes. Estudios han comprobado que más del $50 \%$ de la población laboral ha tenido en algún momento de su vida dolor en la espalda. Y esto se debe principalmente a la exposición de riesgos ergonómicos.

Que según la Organización Internacional del Trabajo la prevalencia mundial de enfermedades ocupaciones en especial las patologías ergonómicas ocupan el primer lugar siendo de gran preocupación para las empresas hoy en día. Cabe destacar que por cada 40 millones de enfermedades ocupacionales el $35 \%$ de tornan crónica, el $10 \%$ generan incapacidad permanente y el $1 \%$ causan la muerte.
Es por ello que la no existencia de una evaluación sobre riesgos ergonómicos en los docentes de la Universidad Técnica Estatal de Quevedo que realizan actividades de teletrabajo y por el desconocimiento de adoptar las posturas adecuadas y condiciones idóneas, esto conllevará a la presencia de enfermedades profesionales por riesgos ergonómicos, los cuales ocupan el primer lugar de prevalencia mundial de enfermedades ocupacionales.

Por todo lo expuesto se hace imprescindible generar una evaluación ergonómica basada en la evaluación del puesto y mediciones técnicas, que permita evaluar los riesgos ergonómicos en los docentes y que a la vez por su exposición generen enfermedades ocupacionales debido a que su prevalencia mundial por patologías ergonómicas ocupan el primer lugar tornándose el $35 \%$ en enfermedades crónicas; evidentemente el aporte de este trabajo estará enfocado a proponer alternativas que garanticen mejorar las condiciones físicas de trabajo y que por ende permitan mantener una salud idónea en los docentes de la institución.

Este trabajo se realizó mediante la identificación de los riesgos ergonómicos inherentes a las posturas que adoptan actualmente los docentes mediante la modalidad del teletrabajo, además se obtuvieron datos concretos mediante la 
aplicación del método ROSA el cual permitió proponer un plan de acción para mitigar los riesgos ergonómicos identificados en los docentes en la modalidad virtual.

\section{MARCO TEÓRICO.}

\subsection{Teletrabajo}

El teletrabajo es una forma flexible de organización del trabajo, que consiste en el desempeño de la actividad profesional sin la presencia física del trabajador en la empresa durante una parte importante de su horario laboral, por una o más jornadas laborales. Ese trabajo a distancia debe realizarse aplicando buenas prácticas y seguridad en el teletrabajo. (Barbosa, 2013)

\subsection{Ergonomía}

Para Wisner (1973), es el conjunto de conocimientos científicos relativos al hombre y necesarios para concebir útiles, máquinas y dispositivos que puedan ser utilizados con la máxima eficacia, seguridad y confort. (Mondelo, 2019)

\subsection{Dimensiones del puesto}

Las posturas y los movimientos naturales son indispensables para un trabajo eficaz, es importante que el puesto de trabajo se adapte a las dimensiones corporales del operario. (Nusshold, P., 2018). Para establecer las dimensiones esenciales de un puesto de trabajo de oficina, tendremos en cuenta los criterios siguientes: Altura del plano de trabajo, espacio reservado para las piernas y zonas de alcance óptimas del área de trabajo. (Instituto Nacional de Higiene y Seguridad en el Trabajo, 1983)

\subsection{Enfermedades profesionales $\mathbf{u}$ ocupacionales}

Son afecciones crónicas, causadas de una manera directa por el ejercicio de la profesión u ocupación que realiza el trabajador y como resultado de la exposición a factores de riesgos, que producen $\mathrm{o}$ no incapacidad laboral. (Instituto Ecuatoriano de Seguridad Social, 2017)

\subsection{Desorden musculoesqueléticos.}

Son desórdenes relacionados con el trabajo incluyendo grupos de condiciones como nervios, tendones, músculos, y estructuras de apoyo como los discos intervertebrales. (OMS, 2018)

\subsection{Lesión musculoesquelética}

Son daños que afectan a los músculos, tendones, huesos, ligamentos o discos intervertebrales. (OMS, 2018)

\subsection{Método ROSA}

El método ROSA calcula la desviación existente entre las características del puesto evaluado y las de un puesto de 
oficina de características ideales. Para ello se emplean diagramas de puntuación que asignan una puntuación a cada uno de los elementos del puesto: silla, pantalla, teclado, mouse y te léfono. (Ergonautas, 2020)

\subsubsection{Aplicación del método}

Obtenidos los datos necesarios tras la observación del puesto se puntúan los diferentes elementos empleando los diagramas de valoración y se emplean las tablas del método para obtener las puntuaciones parciales y la puntuación final.

\subsection{Puntuación de la Silla}

Se comienza obteniendo la Puntuación de la Silla. Para ello es necesario obtener previamente las puntuaciones de la Altura del Asiento, la Profundidad del Asiento, los Reposabrazos y el Respaldo. La puntuación del elemento (que oscilará generalmente entre 1 y 2 o 3 puntos), y determinadas circunstancias que pueden incrementar la puntuación obtenida. Por ejemplo, si el asiento está muy bajo provocando que el ángulo entre el muslo y la pantorrilla sea inferior a $90^{\circ}$ la puntuación de la Altura del Asiento es 2. Si además ocurre que no hay espacio suficiente para las piernas bajo la mesa, la puntuación será incrementada en un punto, resultando una puntuación para la Altura del Asiento de 3. Si además la altura del asiento no fuera regulable la puntuación final sería 4 .
1. Puntuación de la Altura del Asiento

2. Puntuación de la Profundidad del Asiento

3. Puntuación de los Reposabrazos

4. Puntuación del Respaldo

5. Puntuación del tiempo de uso.

\subsection{Puntuación de la Pantalla y los Periféricos}

Es necesario obtener previamente las puntuaciones de la Pantalla, del Teléfono, del Mouse y del Teclado. A diferencia de la puntuación de la silla, la puntuación de la pantalla y los periféricos debe incluir la puntuación por el tiempo de uso. Por ejemplo, la Puntuación de la Pantalla más la puntuación debida al tiempo de uso del monitor obtenida. En este caso, la puntuación por tiempo de uso dependerá del tiempo que el trabajador emplee la pantalla en su jornada. De la misma forma se obtendrán las puntuaciones del resto de elementos: añadiendo la puntuación por tiempo de uso de cada elemento a las obtenidas en los diagramas de valoración.

1. Puntuación de la Pantalla

2. Puntuación del Teléfono

3. Puntuación del Mouse

4. Puntuación del Teclado

\subsection{Nivel de actuación}

El valor de la puntuación ROSA puede oscilar entre 1 y 10, siendo más grande cuanto mayor es el riesgo para la persona que ocupa el puesto. La siguiente tabla 
muestra los Niveles de Actuación según la puntuación final ROSA.

Tabla 1. Niveles de riesgo y actuación (Levels of risk and action)

\begin{tabular}{cccl}
\hline Puntuación & Riesgo & Nivel & \multicolumn{1}{c}{$\begin{array}{c}\text { Actuación } \\
\text { No es necesaria actuación. }\end{array}$} \\
\hline 1 & Inapreciable & 0 & $\begin{array}{l}\text { Pueden mejorarse algunos } \\
\text { elementos del puesto. }\end{array}$ \\
\hline $5-3-4$ & Alto & 1 & $\begin{array}{l}\text { Es necesaria la actuación. } \\
6-7-8\end{array}$ \\
\hline $9-10$ & Muyalto & 3 & $\begin{array}{l}\text { Es necesaria la actuación } \\
\text { cuanto antes. }\end{array}$ \\
\hline
\end{tabular}

Fuente: Ergoniza

\section{METODOLOGÍA}

\subsection{Localización}

El presente estudio de investigación se desarrolló en los espacios utilizados para realizar su trabajo por los docentes en su hogar debido a la modalidad en línea; cabe destacar que los docentes pertenecen al Campus Universitario "Manuel Haz Álvarez" ubicado en el cantón Quevedo, avenida Quito, Km 1.5 vía Sto. Domingo de la provincia de Los Ríos.

\subsection{Población}

El universo sujeto a estudio de investigación corresponde a los 157 docentes, que laboran en la universidad el cual imparten cátedras a los estudiantes de las diferentes facultades que conforman la institución.

\subsection{Muestra}

Para encontrar el tamaño de la muestra se conoce que la institución cuenta con un universo objetivo de 366 , donde utilizó un nivel de confianza del $95 \%$ con un nivel de significancia del $5 \%$ y una varianza del 0.5 , y se aplica la siguiente ecuación:

$$
=\frac{\mathrm{N} \sigma^{2} \mathrm{Z}^{2}}{(\mathrm{~N}-1) \mathrm{e}^{2}+\sigma^{2} \mathrm{Z}^{2}}
$$

Descripción de la ecuación

Tabla 2. Descripción de la ecuación (Description of the equation)

\begin{tabular}{cl}
\hline Símbolo & \multicolumn{1}{c}{ Descripción } \\
\hline $\mathbf{n}$ & Tamaño de la muestra \\
\hline $\mathbf{N}$ & Universo objetivo \\
\hline $\mathbf{e}$ & Error o nivel de significancia \\
$\mathbf{Z}$ & Nivel de confianza \\
\hline
\end{tabular}


$\boldsymbol{\sigma}$

Varianza

Fuente: Elaboración propia

\subsection{Tipo de investigación}

Para la evaluación de los riesgos ergonómicos en los docentes que realizan teletrabajo en la Universidad Técnica Estatal de Quevedo se utilizó:

Investigación exploratoria: Se utilizó para conocer información en cuanto al tema. Debido a que existen pocos trabajos de investigación o estudios de los riesgos ergonómicos en docentes que realizan teletrabajo.

Investigación descriptiva: Mediante esta investigación se analizó los efectos concernientes a riesgos ergonómicos ocasionados por la actividad de teletrabajo que realizan los docentes.

Investigación bibliográfica documental: Consistió en la búsqueda de información en investigaciones, libros, páginas de internet, normativas, reglamentos, los cuales son de fundamentos para el análisis y discusión de los resultados obtenidos.

\subsection{Métodos de investigación}

\subsubsection{Método inductivo}

Mediante este método que va de lo particular a lo general permitió conocer los problemas de los riesgos ergonómicos en los docentes de la institución al realizar teletrabajo y de esta manera poder comparar con otros estudios realizados.

\subsubsection{Método deductivo}

Se utilizó para identificar las limitaciones que se genera en la institución en cuanto al control de exposición a los riesgos ergonómicos, realizando una evaluación y control en el puesto de trabajo de los docentes para finalmente poder resolver el problema general del estudio planteado.

\subsubsection{Método analítico}

Mediante la utilización del método ROSA elaborado por Ergonautas permitió evaluar las posturas adoptadas por los docentes, el cual estimará los niveles de riesgos y actuación mediante el análisis de datos para posterior adoptar medidas preventivas.

\subsection{Fuentes de recopilación de información}

Fuentes primarias: Ayudó a proporcionar información a primera instancia, pues se indago la investigación, mediante encuesta realizada a los docentes que laboran en la institución.

Fuentes secundarias: Mediante esta fuente se procedió a recopilar información de tesis similares, artículos científicos, investigaciones realizada a la gestión técnica de los riesgos ergonómicos.

\subsection{Diseño de la investigación.}

Esta investigación es considerada causi experimental por que se realizó una 
evaluación ergonómica a un grupo de docentes de institución para determinar el nivel de riesgo y vulnerabilidad ante dicha modalidad de trabajo.

\subsection{Instrumentos de la investigación}

Para obtener información confiable que permita conocer y analizar las condiciones de los docentes de la UTEQ, se utilizó:

Encuesta: Para ello se realizó un cuestionario que ayudó a cumplir parte formulación de los objetivos, y que fue aplicada a la muestra para evaluar la percepción de riesgos ergonómicos, y poder aplicar medidas y estrategias que garanticen la seguridad y confort de los docentes.
Fotos: Mediante su utilización permitió ingresar en el software datos específicos para ayudar a determinar resultados en cuanto al nivel de riesgo y actuación.

\subsection{Tratamiento de los datos}

Para la evaluación de los riesgos ergonómicos en docentes que realizan la modalidad de teletrabajo se aplicó el método ROSA y un cuestionario, la tabulación de datos se realizó mediante el programa estadístico combinado excel y SPSS, el cual permitió registrar información e interpretar de manera más rápida y confiable.

\subsection{Recursos humanos $y$ materiales.}

Para esta investigación fue necesaria la utilización de:

Tabla 3. Recursos humanos y materiales (Human and material resources)

\begin{tabular}{ll}
\hline \multicolumn{1}{c}{ Recursos } & \multicolumn{1}{c}{ Descripción } \\
\hline \multirow{3}{*}{ Humanos } & Rector de la UTEQ \\
\cline { 2 - 2 } & Directora de Talento Humano UTEQ \\
\hline & $\begin{array}{l}\text { Docentes de la Universidad Técnica } \\
\text { Estatal de Quevedo }\end{array}$ \\
\hline Libros & Revistas \\
\hline Resma de papel \\
\hline Computadora \\
\hline Impresora multifunciones \\
\hline Internet \\
Esferos \\
\hline Software Ergoniza \\
Fuente: Elaboración Propia
\end{tabular}




\section{RESULTADOS Y DISCUSIÓN}

Al aplicar el método de evaluación ROSA se identifica en la población resultados que están relacionados al disconfort en el puesto de trabajo, para lo cual los docentes evaluados se observaron en la realización de su actividad mediante la encuesta y como consecuencia también se consideró evaluar las posturas de trabajo como son el uso de portátiles y el uso de ratón, pero englobándolas en una sola evaluación bajo este método, teniendo así los siguientes resultados expresados en la tabulación de la tabla de información.

\subsection{Nivel de actuación y riesgo}

En la (tabla 4), detalla que mediante la evaluación del método Rosa se logró identificar que la mitad de la población tiene un riesgo muy alto lo que es necesario una actuación cuanto antes debido a las condiciones que se encuentra laborando no son las idóneas ; mientras, una parte considerable presenta un nivel de riesgo alto considerando una actuación necesaria y un pequeño grupo laboran en condiciones idóneas ubicándose en un nivel de riesgo mejorable lo que indica que pueden mejorarse algunos elementos del puesto.

Tabla 4. Nivel de actuación y riesgo (Levels of risk and action)

\begin{tabular}{|c|c|c|c|c|}
\hline \multirow[b]{2}{*}{ Nivel de actuación } & \multicolumn{3}{|c|}{ Riesgo } & \multirow{2}{*}{$\begin{array}{c}\text { Total } \\
\text { general }\end{array}$} \\
\hline & $\begin{array}{l}\text { Mejorable } \\
\text { (1) }\end{array}$ & $\begin{array}{l}\text { Alto } \\
(2)\end{array}$ & $\begin{array}{l}\text { Muy alto } \\
\text { (3) }\end{array}$ & \\
\hline $\begin{array}{l}\text { Pueden mejorarse algunos } \\
\text { elementos del puesto }\end{array}$ & $6 \%$ & - & - & $6 \%$ \\
\hline Es necesaria actuación & - & $44 \%$ & - & $44 \%$ \\
\hline $\begin{array}{l}\text { Es necesaria actuación } \\
\text { cuanto antes }\end{array}$ & - & - & $50 \%$ & $50 \%$ \\
\hline Total general & $6 \%$ & $44 \%$ & $50 \%$ & $100 \%$ \\
\hline
\end{tabular}

Fuente: Elaboración propia

\subsection{Nivel de riesgo y género}

En la (tabla 5), se muestra que del total de la población según el género representa al sexo masculino con un nivel de riesgo alto y muy alto sucesivamente; lo que significa que tienen mayor incidencia de riesgo ergonómico se encuentran en las personas de esta categoría, mientras que una pequeña parte pertenece al género femenino con un riesgo muy alto y alto respectivamente; teniendo en cuenta que 
existe una pequeña población que de riesgo mejorable. pertenecen al sexo masculino con un nivel

Tabla5. Nivel de riesgo y género (Level of risk and gender)

\begin{tabular}{|c|c|c|c|c|}
\hline \multirow{2}{*}{\multicolumn{2}{|c|}{ Nivel de riesgo }} & \multicolumn{2}{|c|}{ Género } & \multirow{3}{*}{$\begin{array}{c}\text { Total } \\
\text { general } \\
6 \%\end{array}$} \\
\hline & & \multirow{2}{*}{$\begin{array}{c}\text { Masculino } \\
6 \%\end{array}$} & \multirow{2}{*}{$\begin{array}{c}\text { Femenino } \\
-\end{array}$} & \\
\hline Mejorable & $6 \%$ & & & \\
\hline Alto & $44 \%$ & $38 \%$ & $6 \%$ & $44 \%$ \\
\hline Muy alto & $50 \%$ & $44 \%$ & $6 \%$ & $50 \%$ \\
\hline \multicolumn{2}{|c|}{ Total general } & $88 \%$ & $13 \%$ & $100 \%$ \\
\hline
\end{tabular}

\subsection{Nivel de riesgo y años laborales en la UTEQ}

En la (tabla 6), se detalla que según los años de laborales las personas entre 0 a 5 años tiene un nivel de riesgo alto y muy alto; es decir, el riesgo ergonómico está más frecuente, sin embargo, hay que tener en cuenta que para los años de 6 en adelante tienen menos problemas de riesgos ergonómicos esto puede ser debido a que cuentan con condiciones satisfactorias para realizar su trabajo, es decir que el riesgo disminuye pero que se debe tomar medidas correctivas para minimizar el riesgo inherente a la actividad.

\begin{tabular}{|c|c|c|c|c|c|}
\hline \multicolumn{2}{|c|}{ Riesgo } & \multicolumn{3}{|c|}{ Años laborales en la UTEQ } & \multirow{2}{*}{$\begin{array}{c}\text { Total } \\
\text { general }\end{array}$} \\
\hline & & $0-5$ & $6-10$ & Más de 10 & \\
\hline Mejorable & $6 \%$ & $6 \%$ & - & - & $6 \%$ \\
\hline Alto & $44 \%$ & $19 \%$ & $13 \%$ & $13 \%$ & $44 \%$ \\
\hline Muy alto & $50 \%$ & $19 \%$ & $19 \%$ & $13 \%$ & $50 \%$ \\
\hline \multicolumn{2}{|c|}{ Total general } & $44 \%$ & $31 \%$ & $25 \%$ & 160 \\
\hline
\end{tabular}

\subsection{Nivel de riesgo y equipos para realizar su trabajo}

En la (tabla 7), se muestra como existe una correlación que las personas con un nivel de riesgo muy alto y alto son aquellas que utilizan laptop y representan la gran mayoría de la población lo cual indica que se deben tomar acciones para minimizar el nivel de riesgo; es decir que se debe al no constar con los materiales y periféricos esenciales para realizar sus actividades 
cotidianas.

Tabla 7. Nivel de riesgo y equipos para realizar su trabajo (Level of risk and equipment to get your work done)

\begin{tabular}{|c|c|c|c|c|}
\hline \multirow{2}{*}{\multicolumn{2}{|c|}{ Nivel de Riesgo }} & \multicolumn{2}{|c|}{$\begin{array}{c}\text { Equipos para realizar su } \\
\text { trabajo }\end{array}$} & \multirow{2}{*}{$\begin{array}{c}\text { Total } \\
\text { general }\end{array}$} \\
\hline & & $\begin{array}{l}\text { Computadora } \\
\text { escritorio }\end{array}$ & Laptop & \\
\hline Mejorable & $6 \%$ & $6 \%$ & - & $6 \%$ \\
\hline Alto & $44 \%$ & $6 \%$ & $38 \%$ & $44 \%$ \\
\hline Muy alto & $50 \%$ & $6 \%$ & $44 \%$ & $50 \%$ \\
\hline \multicolumn{2}{|c|}{ Total general } & $18 \%$ & $82 \%$ & $100 \%$ \\
\hline
\end{tabular}

\subsection{Nivel de riesgo y percepción de la silla para realizar la actividad}

En la (tabla 8), se puede observar que existe una fuerte relación en cuanto a la percepción de la silla y el nivel de riesgo, debido a que la población de riesgo y muy alto presenta incomodidad con respecto a la silla para realizar teletrabajo; esto se debe a que utilizan las sillas de origen casero y no una que se ajuste a las condiciones antropométricas del cuerpo y de esta manera evitar dolencias en la zona lumbar.

Tabla 8. Nivel de riesgo y percepción de la silla para realizar la actividad (Level of risk and perception of the chair to perform the Activity)

\begin{tabular}{ccccc}
\multirow{2}{*}{ Riesgo } & \multicolumn{2}{c}{ Percepción de la silla } & \multirow{2}{*}{$\begin{array}{c}\text { Total } \\
\text { general }\end{array}$} \\
\cline { 3 - 4 } Mejorable & $6 \%$ & $\begin{array}{c}\text { Poco } \\
\text { incomodo }\end{array}$ & $\begin{array}{c}\text { Muy } \\
\text { incómodo }\end{array}$ & \\
Alto & $44 \%$ & $19 \%$ & - & $6 \%$ \\
Muy alto & $50 \%$ & $19 \%$ & $25 \%$ & $44 \%$ \\
\multicolumn{2}{c}{ Total general } & $44 \%$ & $31 \%$ & $50 \%$ \\
\multicolumn{3}{c}{ Fuente: Elaboración propia } \\
\hline
\end{tabular}

\subsection{Nivel de riesgo y factores ambientales}

En la (tabla 9), se muestra que los docentes tanto en la iluminación y ruido presentan molestias débiles, que van en función del nivel de riesgo; es decir, que la población que representan el nivel de riesgo alto y muy alto están presentando problemas en cuanto a estos factores que al no ser tratados presentaran molestias como: fatiga visual, trastornos del sueño, cansancio. 


\begin{tabular}{|c|c|c|c|c|}
\hline \multirow{2}{*}{\multicolumn{2}{|c|}{ Riesgo }} & \multicolumn{2}{|c|}{ Factores ambientales } & \multirow[b]{2}{*}{$\begin{array}{c}\text { Total } \\
\text { general }\end{array}$} \\
\hline & & $\begin{array}{l}\text { Iluminación } \\
\text { Molestias }\end{array}$ & $\begin{array}{c}\text { Ruido } \\
\text { Molestias }\end{array}$ & \\
\hline Mejorable & $6 \%$ & - & $6 \%$ & $6 \%$ \\
\hline Alto & $44 \%$ & $25 \%$ & $19 \%$ & $44 \%$ \\
\hline Muy alto & $50 \%$ & $22 \%$ & $28 \%$ & $50 \%$ \\
\hline \multicolumn{2}{|c|}{ Total general } & $47 \%$ & $53 \%$ & $100 \%$ \\
\hline
\end{tabular}

\subsection{Nivel de riesgo y antecedentes patologías}

En la (tabla 10), se muestra que la población de riesgo muy alto y alto están tendiente a sufrir lumbalgias, mientras; una pequeña población en ambos niveles de riesgo sufre del síndrome cervical por tensión, esto se debe a las malas posturas que adoptan al realizar sus actividades y al tiempo de exposición al cual se encuentran, y si estas dolencias persisten conllevan a sufrir de enfermedades ocupacionales en la zona lumbar del trabajador.

Tabla 10. Nivel de riesgo y antecedentes patologías (Level of risk and background pathologies)

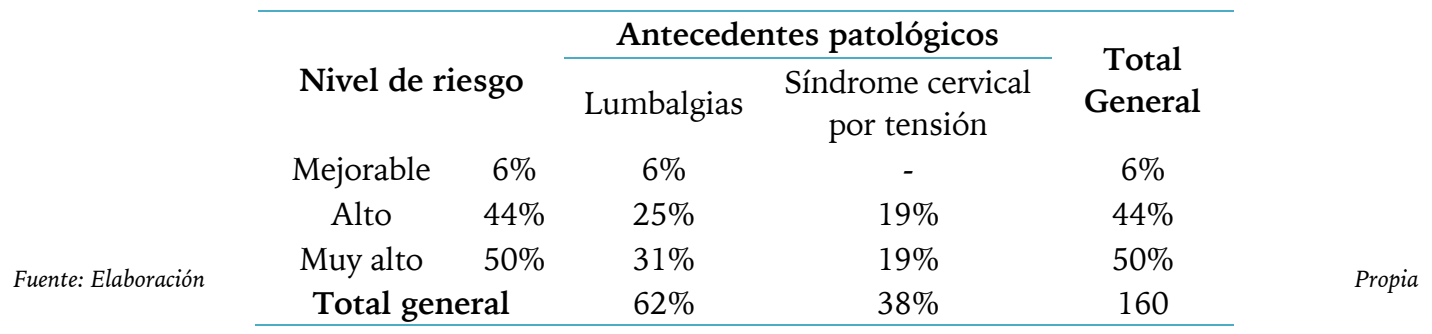

\subsection{Nivel de riesgo y dolor o molestias en el cuerpo}

En la (tabla 11), se muestra que la población sufre dolores y molestias en el cuerpo 1 vez al día en la zona del cuello, espalda alta y espalda baja, los cuales están relacionados a la actividad ejercida en esta modalidad, cuyas partes del cuerpo afectada muestran estrechamente la relación al riesgo ergonómico por posturas inadecuadas adoptadas durante el tiempo de exposición.

Tabla 11. Nivel de riesgo y dolor o molestias en el cuerpo (Level of risk and pain or discomfort in the body) 


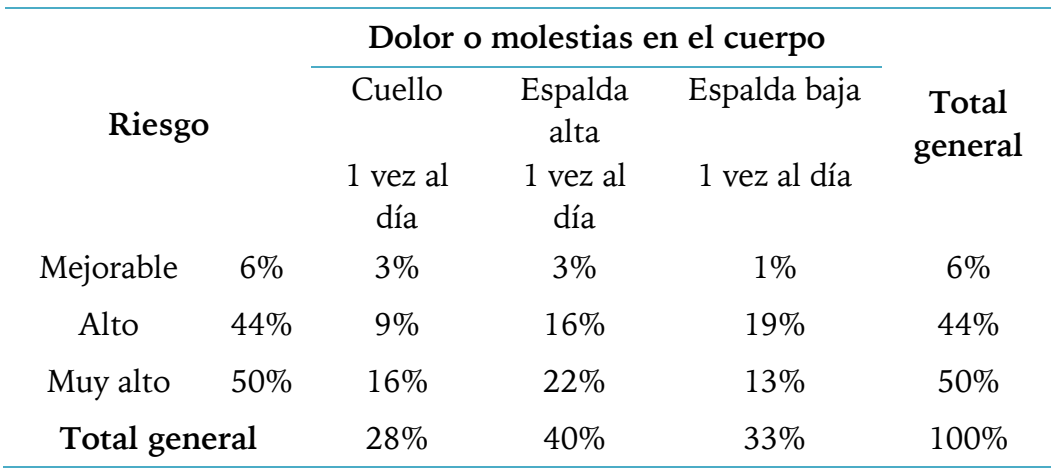

Fuente: Elaboración propia

\section{CONCLUSIONES}

Después de haber aplicado el método ROSA en la muestra seleccionada y cuestionario ergonómico y en correlación al objetivo general planteado cuya finalidad fue identificar los riesgos ergonómicos presentes en la modalidad de teletrabajo se puede concluir lo siguiente:

En cuanto a los resultados de la encuesta ergonómica se identifica que existen dos parámetros que podrían generar mayor riesgo y disconfort en los docentes, los mismos que se relacionan por una parte a accesorios como ratón, teclado y elevador de laptop considerando que los docentes mantienen una interacción continua con equipos portátiles; por otra parte, la silla que no está relacionada con las características antropométricas de los mismos.

En cuanto al método ROSA los resultados evidencian que para el cincuenta por ciento de la población el nivel de riesgo obtenido es muy alto, y que trabajando bajo esas medidas adoptadas será necesario un rediseño inmediato del puesto de trabajo ya que las actuales podrían evidenciar daños a la salud de los docentes.

Se elaboró un plan de acción con la finalidad de minimizar y controlar los riesgos identificados en las actividades realizadas en la modalidad de teletrabajo.

\section{REFERENCIAS}

\section{Libro}

\subsection{Libro impreso}

[1]. Buira, J. (2012). El teletrabajo entre el mito y la realidad. Barcelona, España: UOC.

[2]. Barbosa, V. K. (2013). Teletrabajo. Liderar y trabajar en equipos a distancia. Buenos Aires: DUNKEN.

[3]. Díaz, J. M. (2015). Seguridad e Higiene del Trabajo, Tecnicas de Prevención de Riesgos Laborales. Madrid, España: Tebal, S.L.

[4]. Koenes, A. (2012). Plan de 
negocios. Madrid, España: DIAZ DE SANTOS, S.A.

[5]. Mondelo, P. (2019). Ergonomía 1 Fundamentos. Barcelona: Mutua Universal, Edicions UPC.

[6]. Urquijo, J. I. (2004). Teoria de las relaciones Sindicato Gerenciales. Carácas, Venezuela: Universidad Católica Andrés Bello.(Maestria)

\section{Tesis}

[7]. Castellano, M. B. (Agosto de 2018). Evaluación ergonómica de personal administrativo que realiza teletrabajo, en una compañía comercializadora de productos alimenticios. Obtenido de https://repositorio.uisek.edu.ec/ha ndle/123456789/3131 (Maestría)

\section{Página web}

[8]. OMS. (2018). Prevención de transtornos músculo-esqueléticos, riesgos en el trabajo. Obtenido de https://www.who.int/es

[9]. Ergonautas. (2020). Método de ROSA. Obtenido de https://www.ergonautas.upv.es/me todos/rosa/rosa-ayuda.php

[10]. Instituto Nacional de Higiene y Seguridad en el Trabajo. (1983). NTP 242: Ergonomía: análisis ergonómico de los espacios de. Obtenido de https://www.cnae.com/ficheros/fil es/prl/ntp_242.pdf

[11]. Instituto Ecuatoriano de Seguridad Social. (17 de Junio de 2017). Resolución IESS 513 Reglamento del Seguro General de Riesgos del Trabajo. Resolución IESS 513 Reglamento del Seguro General de Riesgos del Trabajo. Quito, Ecuador: Edición especial 623. 